\title{
Cost-effectiveness of Bariatric Surgery
}

\author{
Matthew L. Maciejewski, PhD and David E. Arterburn, MD, MPH \\ Center for Health Services Research in Primary Care, Durham VA Medical Center, Durham, \\ North Carolina (Maciejewski); Division of General Internal Medicine, Department of Medicine, \\ Duke University, Durham, North Carolina (Maciejewski); Group Health Research Institute, \\ Seattle, Washington (Arterburn); Department of Medicine, Division of General Internal Medicine, \\ University of Washington, Seattle (Arterburn)
}

\section{Abstract \\ JAMA SURGERY - Impact of Bariatric Surgery on Health Care Costs of Obese Persons: A 6- Year Follow-up of Surgical and Comparison Cohorts Using Health Plan Data \\ Jonathan P. Weiner, DrPH; Suzanne M. Goodwin, PhD; Hsien-Yen Chang, PhD, MHS; Shari D. Bolen, MD, MPH; Thomas M. Richards, MSEE; Roger A. Johns, MD, MHS; Soyal R. Momin, MS, MBA; Jeanne M. Clark, MD, MPH}

IMPORTANCE-Bariatric surgery is a well-documented treatment for obesity, but there are uncertainties about the degree to which such surgery is associated with health care cost reductions that are sustained over time.

OBJECTIVE-To provide a comprehensive, multiyear analysis of health care costs by type of procedure within a large cohort of privately insured persons who underwent bariatric surgery compared with a matched nonsurgical cohort.

DESIGN-Longitudinal analysis of 2002-2008 claims data comparing a bariatric surgery cohort with a matched nonsurgical cohort.

SETTING-Seven BlueCross BlueShield health insurance plans with a total enrollment of more than 18 million persons.

PARTICIPANTS-A total of 29820 plan members who underwent bariatric surgery between January 1, 2002, and December 31, 2008, and a 1:1 matched comparison group of persons not undergoing surgery but with diagnoses closely associated with obesity.

MAIN-Outcome Measures Standardized costs (overall and by type of care) and adjusted ratios of the surgical group's costs relative to those of the comparison group.

Corresponding Author: Matthew L. Maciejewski, PhD, Center for Health Services Research in Primary Care (152), Durham VA Medical Center, 411 W Chapel Hill St, Ste 600, Durham, NC 27705, matthew.maciejewski@va.gov.

Disclaimer: The opinions expressed are those of the authors and not necessarily those of the Department of Veterans Affairs, the US government, Duke University, the Group Health Research Institute, or the University of Washington.

Additional Contributions: Chris Tachibana, $\mathrm{PhD}$, and Emily Westbrook, BA, Group Health Research Institute, provided editorial assistance. They were not compensated besides their salaries.

Conflict of Interest Disclosures: Both authors have completed and submitted the ICMJE Form for Disclosure of Potential Conflicts of Interest. Dr Maciejewski reported having received consultation funds from Daichi Sankyo, Takeda Pharmaceuticals, Novartis, ResDAC at the University of Minnesota, and the Surgical Review Corporation and owning stock in Amgen because of his spouse's employment. Dr Arterburn reported having received research funding and salary support as a medical editor for the not-for profit (501[3]c) Informed Medical Decisions Foundation (http://www.informedmedicaldecisions.org), which develops content for patient education programs. The foundation has an arrangement with a for-profit company, Health Dialog, to coproduce and market these programs to health care organizations. 
RESULTS-Total costs were greater in the bariatric surgery group during the second and third years following surgery but were similar in the later years. However, the bariatric group's prescription and office visit costs were lower and their inpatient costs were higher. Those undergoing laparoscopic surgery had lower costs in the first few years after surgery, but these differences did not persist.

CONCLUSIONS AND RELEVANCE-Bariatric surgery does not reduce overall health care costs in the long term. Also, there is no evidence that any one type of surgery is more likely to reduce long-term health care costs. To assess the value of bariatric surgery, future studies should focus on the potential benefit of improved health and well-being of persons undergoing the procedure rather than on cost savings.

JAMA Surg. 2013;148(6):555-562.

Bariatric surgery is probably more efficacious than medical or lifestyle intervention for long-term weight loss and remission of diabetes. The ability of bariatric surgery to reduce expenditures sufficiently to achieve cost savings remains hotly debated, and in 2 early studies, it appeared cost-saving over a relatively short period of time. ${ }^{1,2}$ More recent studies, 3,4 including an analysis of 29820 BlueCross BlueShield enrollees published in the June issue of JAMA Surgery, ${ }^{5}$ show no evidence of cost savings. These results are consistent with prior cost-effectiveness evaluations ${ }^{6,7}$ that demonstrated cost-effectiveness—but not cost savings-for bariatric procedures compared with usual medical care or intensive lifestyle interventions. Still, a critical policy question remains unanswered: does bariatric surgery need to be cost-effective (ie, more effective but more costly than usual care), or does it need to achieve the higher standard of cost savings (ie, more effective and less costly than usual care) to justify broader insurance coverage?

Because bariatric surgery is so expensive, it is unlikely to achieve a cost-saving threshold for most eligible patients. Roux-en-Y gastric bypass (RYGB) costs $\$ 25000$ to $\$ 30000$ for the surgical admission (including the cost of the procedure and immediate preoperative, intraoperative, and postoperative services), and laparoscopic adjustable gastric banding (LAGB) costs $\$ 15000$ to $\$ 20000 .{ }^{5}$ Annual health care costs for patients with a body mass index (BMI) of 35 or greater are $\$ 3000$ to $\$ 10000$ per year. ${ }^{4,5}$ Even if total expenditures are reduced $50 \%$ after surgery, it may take up to 20 years to achieve cost neutrality. There is a need to identify whether certain bariatric procedures or patient subgroups have greater potential for health improvements and cost savings to inform insurance coverage decisions about bariatric procedures.

\section{Are Certain Procedures More Likely to Provide Cost Savings?}

The recent JAMA Surgery article addresses, in a limited way, whether some bariatric procedures might have greater potential for cost savings. ${ }^{5}$ Inpatient and total expenditures were lower 2 years after laparoscopic RYGB or LAGB compared with open RYGB, but expenditures for laparoscopic and open RYGB became statistically similar by year 6 . None of the 3678 patients undergoing LAGB were followed up long enough to report 6-year expenditures. More follow-up is needed.

The lack of cost savings for RYGB and LAGB is unsurprising because RYGB is the most invasive and complicated procedure and is associated with the highest early complication rates. Three articles, ${ }^{3-5}$ using different but equally rigorous methods to construct a matched cohort of nonsurgical controls, suggest that costs after RYGB are not significantly reduced because inpatient costs remain high for surgical patients, attributed at least in part to the higher complication rates. LAGB has also been associated with higher inpatient costs due to high rates of reoperations and band removals. ${ }^{8,9}$ Newer procedures, such as sleeve 
gastrectomy, might yield cost savings due to relatively low complication rates and early evidence of clinical outcomes that are comparable with RYGB in some patient populations. ${ }^{10}$ However, sleeve gastrectomy has yet to undergo long-term economic evaluation.

\section{Are Certain Patient Characteristics Associated With Greater Cost Savings?}

No prior cost studies and few cost-effectiveness analyses have examined whether certain patient subgroups experience greater expenditure reductions than others. Answering this question is critical to establishing the economic case for bariatric surgery. More research is needed on the heterogeneity of treatment associations in large cohorts such as the JAMA Surgery study ${ }^{5}$ because an average null association may mask a protective effect for a subgroup of surgical patients (eg, those with type 2 diabetes). This work would be facilitated by standardized measurement and collection of pre-operative and postoperative behavioral, psychological, and motivational data with valid instruments.

\section{Conclusions}

The JAMA Surgery study by Weiner et $\mathrm{al}^{5}$ adds to a limited evidence base showing that bariatric surgery may be cost-effective—but not cost-saving—because postsurgical costs are similar to costs of nonsurgical patients at $3^{4,5}$ to $20^{3}$ years after surgery. The time horizon that is relevant for determining the financial case of any intervention can vary widely among payers in the United States. Given the significant turnover in commercially insured populations, early cost reductions (or cost neutrality) are perhaps more important for payers like BlueCross BlueShield to demonstrate a compelling business case for bariatric surgery. Payers like Medicare or the Veterans Health Administration can accept longer time horizons to achieve health care cost reductions because they are typically responsible for enrollees over many years. For patients and payers, the research community must come together to determine which bariatric procedures for which patient subgroups are most cost-effective because these latest results suggest that the hoped-for cost savings are unlikely to be realized for typical RYGB and LAGB patients.

\section{Acknowledgments}

Funding/Support: Dr Maciejewski is supported by a Research Career Scientist award (RCS 10-391) and a grant (IIR 10-159) from the Department of Veterans Affairs. Dr Arterburn is supported by grants from the National Institutes of Health (R01 DK092317-01 and R01 DK089528-01).

Role of the Sponsor: The Health Services Research and Development Service, Department of Veterans Affairs, had no role in the preparation, review, or approval of the manuscript or decision to submit the manuscript for publication.

\section{References}

1. Cremieux PY, Buchwald H, Shikora SA, Ghosh A, Yang HE, Buessing M. A study on the economic impact of bariatric surgery. Am J Manag Care. 2008; 14(9):589-596. [PubMed: 18778174]

2. Finkelstein EA, Allaire BT, Burgess SM, Hale BC. Financial implications of coverage for laparoscopic adjustable gastric banding. Surg Obes Relat Dis. 2011; 7(3):295-303. [PubMed: 21195677]

3. Neovius M, Narbro K, Keating C, et al. Health care use during 20 years following bariatric surgery. JAMA. 2012; 308(11):1132-1141. [PubMed: 22990272]

4. Maciejewski ML, Livingston EH, Smith VA, Kahwati LC, Henderson WG, Arterburn DE. Health expenditures among high-risk patients after gastric bypass and matched controls. Arch Surg. 2012; 147(7):633-640. [PubMed: 22802057] 
5. Weiner JP, Goodwin SM, Chang H-Y, et al. Impact of bariatric surgery on health care costs of obese persons: a 6-year follow-up of surgical and comparison cohorts using health plan data. JAMA Surg. 2013; 148(6):555-562. [PubMed: 23426865]

6. Picot J, Jones J, Colquitt JL, et al. The clinical effectiveness and cost-effectiveness of bariatric (weight loss) surgery for obesity: a systematic review and economic evaluation. Health Technol Assess. 2009; 13(41):1-190. 215-357.

7. Padwal R, Klarenbach S, Wiebe N, et al. Bariatric surgery: a systematic review of the clinical and economic evidence. J Gen Intern Med. 2011; 26(10):1183-1194. [PubMed: 21538168]

8. Himpens J, Cadière GB, Bazi M, Vouche M, Cadière B, Dapri G. Long-term outcomes of laparoscopic adjustable gastric banding. Arch Surg. 2011; 146(7):802-807. [PubMed: 21422330]

9. Spivak H, Abdelmelek MF, Beltran OR, Ng AW, Kitahama S. Long-term outcomes of laparoscopic adjustable gastric banding and laparoscopic Roux-en-Y gastric bypass in the United States. Surg Endosc. 2012; 26(7):1909-1919. [PubMed: 22219011]

10. Schauer PR, Kashyap SR, Wolski K, et al. Bariatric surgery versus intensive medical therapy in obese patients with diabetes. N Engl J Med. 2012; 366(17):1567-1576. [PubMed: 22449319] 


\section{Box}

\section{Definitions}

\section{Cost-effective}

When an intervention either has equal effectiveness but lower costs as a comparator, or has equal costs and greater effectiveness, such that the incremental cost is below a typical policy threshold (eg, $\$ 50000$ per quality-adjusted life-year).

\section{Cost-saving}

When an intervention is lower cost and more effective compared with a comparator. 